\title{
Controlled ovarian hyperstimulation for fertility preservation in women with breast cancer: Practical issues
}

\author{
So Yun Park, Kyungah Jeong, Eun Hye Cho, Hye Won Chung \\ Department of Obstetrics and Gynecology, Ewha Womans University College of Medicine, Seoul, Korea
}

In Korean women, a westernized lifestyle is associated with an increased risk of breast cancer. Fertility preservation has become an increasingly important issue for women with breast cancer, in accordance with substantial improvements in survival rate after cancer treatment. The methods of controlled ovarian hyperstimulation $(\mathrm{COH})$ for fertility preservation in breast cancer patients have been modified to include aromatase inhibitors to reduce the potential harm associated with increased estradiol levels. Random-start $\mathrm{COH}$ and dual ovarian stimulation are feasible options to reduce the total duration of fertility preservation treatment and to efficiently collect oocytes or embryos. Using a gonadotropin-releasing hormone agonist as a trigger may improve cycle outcomes in breast cancer patients undergoing $\mathrm{COH}$ for fertility preservation. In young breast cancer patients with $B R C A$ mutations, especially $B R C A 1$ mutations, the possibility of diminished ovarian reserve may be considered, although further studies are necessary. Herein, we review the current literature on the practical issues surrounding $\mathrm{COH}$ for fertility preservation in women with breast cancer.

Keywords: Breast neoplasms; Fertility preservation; Ovarian hyperstimulation

\section{Introduction}

Breast cancer is the most common cancer in women, and one in five patients are younger than 45 years old at diagnosis [1]. In light of substantial improvements in cancer treatment, fertility preservation has become an increasing priority for women with breast cancer $[2,3]$. In the United States, the overall 5-year survival rate in women with breast cancer has increased from $74.6 \%$ in $1975-1979$ to $91.0 \%$ in 2007 [4].

A westernized diet, early menarche or late menopause, delayed

Received: February 18, 2020 · Revised: August 10, 2020 • Accepted: August 12, 2020 Corresponding author: Kyungah Jeong

Department of Obstetrics and Gynecology, Ewha Womans University Mokdong Hospital, Ewha Womans University College of Medicine, 1071 Anyangcheon-ro, Yangcheon-gu, Seoul 07985, Korea

Tel: +82-2-2650-2858 Fax: +82-2-2647-9860 E-mail: ogjeong@ewha.ac.kr

This is an Open Access article distributed under the terms of the Creative Commons Attribution Non-Commercial License (http://creativecommons.org/licenses/by-nc/4.0/) which permits unrestricted non-commercial use, distribution, and reproduction in any medium, provided the original work is properly cited. marriage, and having fewer children later in life are all associated with the increasing risk of breast cancer that has been observed among Korean women. Since 1996, the proportion of early-stage breast cancers has significantly increased, and the incidence rate of female breast cancer was 67.2 cases per 100,000 women in 2010 [5]. Breast cancer is currently the second most common cancer in Korean women, after thyroid cancer. The current methods of controlled ovarian hyperstimulation $(\mathrm{COH})$ for fertility preservation in breast cancer patients have been modified. To reduce the risks associated with increased estradiol levels, aromatase inhibitors (Als) are co-administered, unlike in other cancers. In contrast to typical infertility treatment, random-start ovarian stimulation is used to minimize the time typically required for fertility preservation treatment prior to chemotherapy.

The Korean Society for Fertility Preservation has suggested that an appropriate method of fertility preservation should be decided through individual patient counseling. Methods such as the cryopreservation of embryos, oocytes, or ovarian tissue and gonadotro- 
pin-releasing hormone (GnRH) agonist treatment during chemotherapy may be considered, and patients must consult with experts before chemotherapy or any other treatment [6]. Herein, practical concerns related to fertility preservation in women with breast cancer will be discussed. With this review, we aim to provide useful clinical information based on recent studies.

\section{Letrozole administration during ovarian stimulation}

Estrogen exposure is a well-known risk factor for estrogen receptor (ER)-positive breast cancer [7,8]. The role of estrogen in the carcinogenesis of ER-negative breast cancer has yet to be elucidated, and estrogens are thought to have little effect on breast carcinogenesis in ER-negative breast cancer [9]. However, several studies have suggested a possible association between estrogen-dependent reproductive characteristics such as high parity and the incidence of ER-negative breast cancer $[10,11]$. Therefore, treatment with letrozole may be useful even in patients with ER-negative breast cancer.

Letrozole is an Al that prevents estrogen production through competitive, reversible binding to the heme of the cytochrome P450 unit of aromatase [12]. Clinically, $5 \mathrm{mg} /$ day letrozole (Femara; Novartis, East Hanover, NJ, USA) is administered on day 2 or 3 of the patient's cycle. The administration of letrozole is continued daily during $\mathrm{COH}$ and discontinued on the day of the trigger. After oocyte retrieval, letrozole is continued again for approximately 3 to 6 days until estradiol levels decrease to $<50 \mathrm{pg} / \mathrm{mL}$ [13].

Evidence suggests that the administration of letrozole during $\mathrm{COH}$ can significantly reduce peak estradiol concentrations. Peak estradiol concentrations (337-829 pg/mL) were found to be less than 1,000 $\mathrm{pg} / \mathrm{mL}$ when letrozole was commenced on days 2-3 [14-18]. However, Azim et al. [19] reported that anastrozole did not suppress estradiol at its maximum tolerated oral dose of $10 \mathrm{mg} /$ day compared to $5 \mathrm{mg} /$ day of letrozole $(2,515.07 \pm 1,368.52 \mathrm{vs.} 714 \pm 440.83 \mathrm{pg} / \mathrm{mL}$, respectively; $p=0.01$ ), and letrozole was more potent than anastrozole for the suppression of estrogen.

While it potentially avoids the negative effects of estrogens on tumor growth, letrozole prevents the aromatization of androgens to estrogens, which may induce significant changes in the endocrine follicular environment and impact oocyte competence. Several studies have investigated whether the co-administration of letrozole has an impact on oocyte yield. Oktay et al. [16] and Checa Vizcaino et al. [20] both reported that oocyte yield was not significantly affected when letrozole was administered. In contrast, Domingo et al. [17] and Revelli et al. [18] demonstrated a small but significant decrease in oocyte yield. The administration of letrozole with gonadotropins significantly reduced the number of oocytes available for cryostor- age in comparison with the use of gonadotropins alone in breast cancer patients [18]. The triggering of ovulation with a gonadotropin-releasing hormone agonist ( $\mathrm{GnRHa}$ ) has been suggested to help minimize its negative effect [21], and in vitro maturation is a useful strategy to improve the mature oocyte yield in breast cancer patients undergoing ovarian stimulation for fertility preservation [15].

A recent systematic review identified 1,002 records, of which 15 selected studies were included in the final analysis [22]. No evidence was found of a decline in relapse-free survival rates in women with breast cancer who received $\mathrm{COH}$ with the coadministration of letrozole compared with women who did not undergo fertility preservation procedures. Kim et al. [23] reported the largest long-term study, including over 5 years of safety data, regarding the use of $\mathrm{COH}$ with letrozole supplementation for fertility preservation via embryo or oocyte cryopreservation in women with breast cancer. The mean follow-up duration after diagnosis was 5.0 years in the fertility preservation group and 6.9 years among the control patients, who chose not to undergo fertility preservation. In the fertility preservation group, the hazard ratio for recurrence after ovarian stimulation was 0.77 (95\% confidence interval, $0.28-2.13$ ), and survival was not compromised compared with controls ( $p=0.61)$. Neither BRCA gene mutation status $(p=0.57)$ nor whether fertility preservation was undergone before or after breast surgery $(p=0.44)$ significantly affected survival outcomes in the fertility preservation group. None of the tumor characteristics, including ER status, affected the survival rates after $\mathrm{COH}$ with letrozole supplementation [23]. $\mathrm{COH}$ with concurrent use of Als appeared to be a safe fertility preservation option for young women with breast cancer at risk of fertility loss following chemotherapy.

Tamoxifen, sold under the brand name Nolvadex among others, is a selective ER modulator. It is a nonsteroidal triphenylethylene antiestrogen. In breast cancer patients, tamoxifen has been proven to have an excellent suppressive effect on cancer cell proliferation and tumorigenesis. Tamoxifen is often preferred over other treatments, as it does not antagonize endometrial development. Oktay et al. [14] compared tamoxifen and letrozole during $\mathrm{COH}$ for embryo cryopreservation in breast cancer patients. They used $60 \mathrm{mg} /$ day of tamoxifen alone (Tam-in vitro fertilization [IVF]), $60 \mathrm{mg} /$ day of tamoxifen in combination with low-dose follicle-stimulating hormone (FSH; TamFSH-IVF), or $5 \mathrm{mg}$ of letrozole in combination with FSH (letrozole-IVF). The combination of low-dose FSH with tamoxifen (TamFSH-IVF) or letrozole (letrozole-IVF) resulted in higher embryo yields than Tam-IVF. The recurrence rates did not appear to be higher, but the letrozole protocol may be preferred due to lower peak estradiol levels. However, due to insufficient data, conclusions could not be drawn regarding the use of tamoxifen in women with breast cancer during $\mathrm{COH}[20,24]$. Several relevant studies of Al or tamoxifen 
administration during $\mathrm{COH}$ in women with breast cancer are summarized in Table 1.

\section{Random-start ovarian stimulation}

Traditionally, ovarian stimulation is initiated in the early follicular phase within 3 days of the period before dominant follicle selection. The rationale for this timing includes the stimulation of a synchronous cohort of antral follicles recruited during the interfollicular transition and the induction of timely endometrial development to synchronize blastocyst development with the implantation window. However, the synchronization of endometrial development is not required if the aim of the cycle is oocyte or embryo cryopreservation for fertility preservation without fresh embryo transfer (ET) for immediate pregnancy. This is particularly pertinent in cases subject to time constraints, such as those of cancer patients awaiting gonadotoxic chemotherapy.

After an early referral to a fertility specialist, the prompt use of $\mathrm{COH}$ instead of the conventional protocol is very important in all reproductive-aged women undergoing cancer treatment who desire fertility preservation. To provide optimal cancer treatment with minimal delay, random-start ovarian stimulation can be performed. In patients with breast cancer who undergo surgical resection as the first step of cancer therapy, $\mathrm{COH}$ begins immediately for a 4- to 6-week window between surgery and adjuvant chemotherapy. Therefore, the conventional initiation of $\mathrm{COH}$ within 3 days of the period is impossible, and random-start ovarian stimulation can be used at the initial visit. $\mathrm{COH}$ can be initiated before and/or immediately after surgery. Oocyte or embryo cryopreservation can be accomplished with random-start $\mathrm{COH}$ within 2-3 weeks, and patients can proceed subsequently with surgery or additional cancer treatment such as chemotherapy, radiation, and/or hormonal therapy [25].

A GnRH antagonist can be administered to pause the patient's cycle, and this approach has been shown to have no negative impact on embryo quality and to improve synchronous follicular growth in poor responders [26]. The appropriate use of a $\mathrm{GnRH}$ antagonist can accomplish random-start $\mathrm{COH}$ regardless of the point in the menstrual cycle. In a prior study, ovarian stimulation without a GnRH antagonist was initiated if the follicle cohort following the lead follicle was smaller than $12 \mathrm{~mm}$ and remained smaller than $12 \mathrm{~mm}$ prior to a spontaneous luteinizing hormone (LH) surge. After an LH surge, administration of a GnRH antagonist was initiated when the secondary follicle cohort reached $12 \mathrm{~mm}$ to prevent a premature secondary LH surge. If the follicle cohort following the lead follicle reached 12 $\mathrm{mm}$ before the spontaneous $\mathrm{LH}$ surge, pituitary suppression with a $\mathrm{GnRH}$ antagonist was initiated and continued until the triggering of final oocyte maturation. Ovulation was induced with human chori- onic gonadotropin ( $\mathrm{hCG}$ ) or a GnRH agonist when the dominant follicle reached $18 \mathrm{~mm}$ in diameter, and ovarian stimulation was initiated 2-3 days into the luteal phase [27]. To downregulate LH and initiate luteolysis, a GnRH antagonist was administered with recombinant FSH while simultaneously initiating follicular stimulation.

Martinez et al. [28] published the largest study reporting the outcomes of different random-start protocols with and without Als. According to that study, random-start ovarian stimulation provided a significant advantage by decreasing the total time for an IVF cycle, and in emergent settings, ovarian stimulation can be initiated at a random cycle date for fertility preservation without compromising oocyte yield or maturity. Although random-start $\mathrm{COH}$ protocols are efficient in obtaining an appropriate number of mature oocytes/embryos, only a minority of the patients underwent thawing and ET, and not enough reports have been conducted to evaluate the rates of implantation and pregnancy. Nevertheless, comparable pregnancy rates after the transfer of embryos created from donor eggs obtained after luteal-phase start protocols are encouraging.

Danis et al. [29] published a systematic review of 19 studies published between January 2000 and June 2017 to evaluate the utility of random-start ovarian stimulation for fertility preservation. This recent review suggested that random-start stimulation was associated with a reduced time interval between the initiation of ovarian stimulation and oocyte or embryo cryopreservation. The yield of mature oocytes and their potential development into embryos was comparable between the conventional and random-start protocols, although the gonadotropin doses were higher in the random-start protocols.

Therefore, additional clinical studies are necessary to further assess the efficacy of this strategy, especially regarding the rates of clinical pregnancy and live-born infants originating from the use of cryopreserved embryos and oocytes obtained via random-start ovarian stimulation.

\section{Dual ovarian stimulation}

Dual ovarian stimulation consists of two successive instances of ovarian stimulation in the follicular and ensuing luteal phase, with oocyte retrieval at the end of each ovarian stimulation. Evidence is accumulating that follicular- and luteal-phase $\mathrm{COH}$ is feasible in patients with poor prognoses with a reduced ovarian reserve to increase their chances of conceiving in each menstrual cycle [30-32].

Kuang et al. [30] investigated the efficiency of dual ovarian stimulation with a combination of gonadotropins, clomiphene citrate, and Al in the follicular and luteal phases in patients with poor ovarian response. In this pilot study, the first stimulation was conducted using a combination of $25 \mathrm{mg}$ per day of clomiphene citrate starting on 


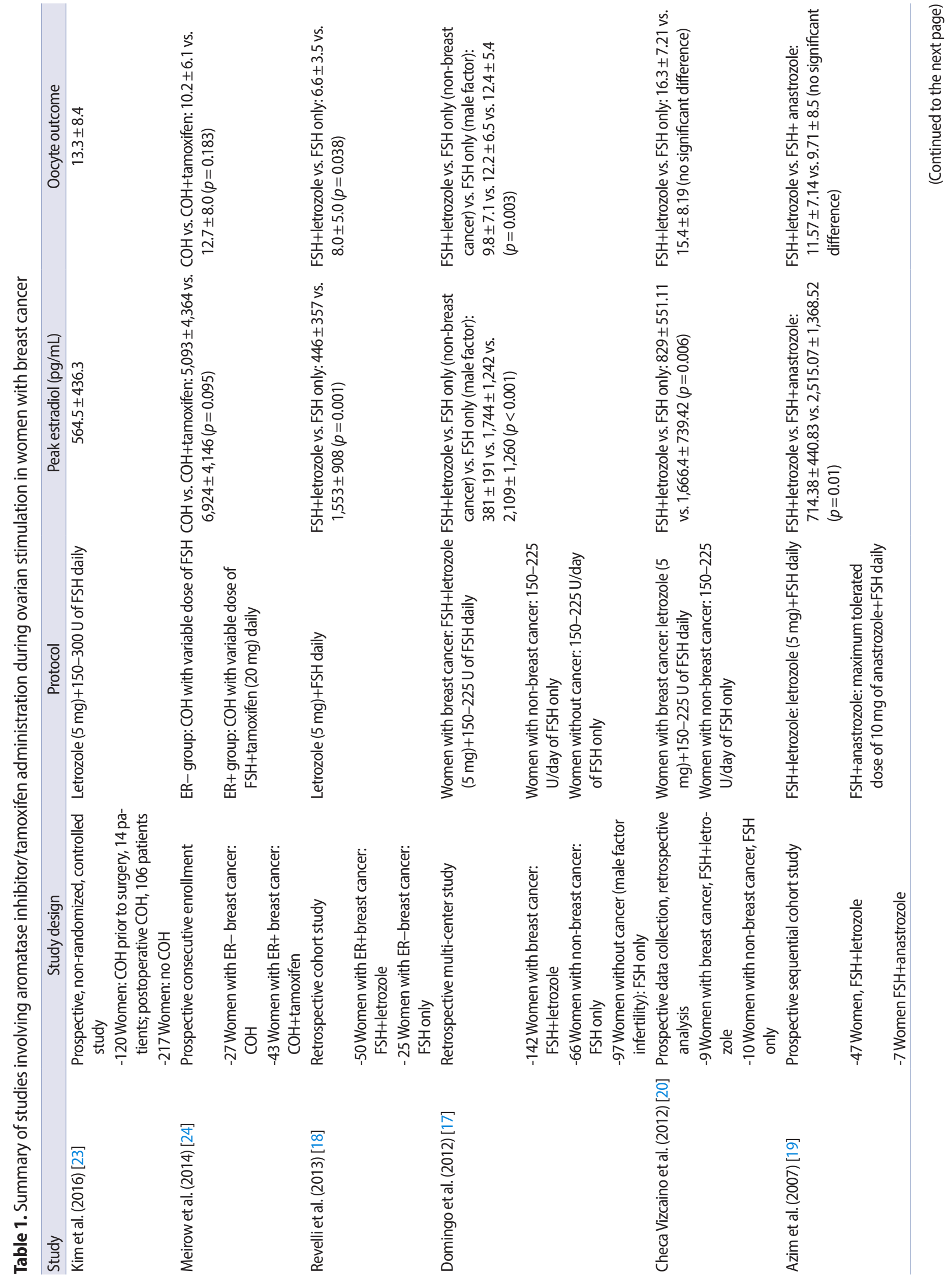




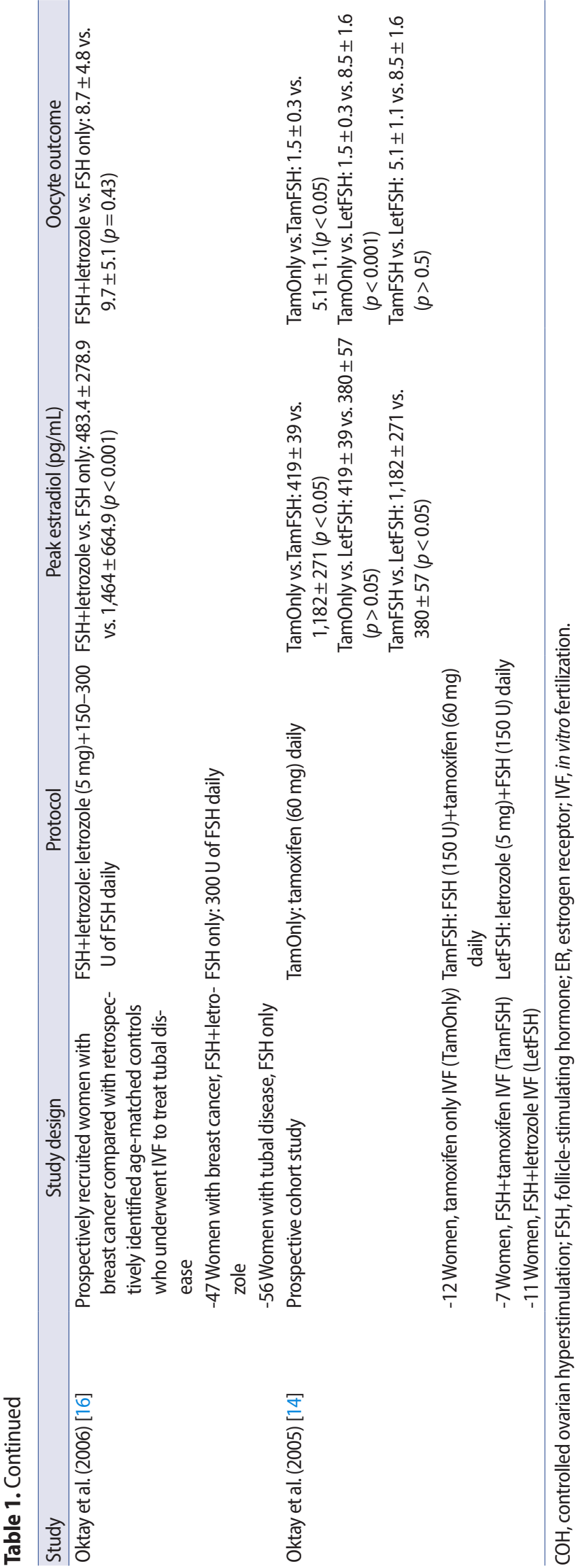

day 3 of the cycle until ovulation was triggered, $2.5 \mathrm{mg}$ of letrozole per day starting on day 3 for a total of 4 days, and $150 \mathrm{IU}$ of human menopausal gonadotropin (hMG) every other day starting on day 6 until the triggering of ovulation. The second stimulation was initiated after the first oocyte retrieval, provided that two or more antral follicles were identified, and it consisted of $2.5 \mathrm{mg}$ of letrozole and $225 \mathrm{IU} /$ day of hMG, which were both administered from the day of retrieval until the second trigger of ovulation. A relatively large dose of hMG was used to perform ovarian stimulation after the first oocyte retrieval due to the ovarian insensitivity to hMG stimulation during the luteal phase relative to the follicular phase. For both the first and second stimulations, final oocyte maturation was induced with a GnRH agonist (triptorelin, 100 $\mathrm{gg}$ ) when follicular maturation was reached. The authors reported similar developmental potential for the antral follicles from the luteal phase compared with those from the follicular phase in terms of mature oocytes, fertilization rate, cleavage rate, and the number of top-quality embryos obtained [30].

In a more recent prospective paired non-inferiority observational study, Ubaldi et al. [31] reported a similar number of euploid blastocysts per metaphase II oocyte injected after follicular- and luteal-phase $\mathrm{COH}$ in the same menstrual cycle. In follicular-phase $\mathrm{COH}$ versus luteal-phase $\mathrm{COH}$, no differences were observed in the number of retrieved cumulus-oocyte complexes ( $5.1 \pm 3.4$ vs. $5.7 \pm 3.3$, respectively), metaphase ll oocytes ( $3.4 \pm 1.9$ vs. $4.1 \pm 2.5$, respectively), biopsied blastocysts (1.2 \pm 1.2 vs. $1.4 \pm 1.7$, respectively), or euploid blastocysts ( $0.6 \pm 0.8$ vs. $0.7 \pm 0.8$, respectively). The types of chromosomal abnormalities observed in aneuploid embryos from the follicular-phase $\mathrm{COH}$ and luteal-phase $\mathrm{COH}$ were also similar, suggesting no impact on the meiotic maturation of oocytes.

Two studies were recently published and reported promising results of double ovarian stimulation [32,33]. Cardoso et al. [33] conducted a retrospective and comparative study of 13 patients who underwent unsuccessful IVF cycles with a conventional antagonist ovarian stimulation protocol and repeated the attempt with a double stimulation protocol. They used the conventional ovarian stimulation antagonist protocol involving a high dose of gonadotropins (225 IU of FSH and $75 \mathrm{IU}$ of hMG), and the triggering was carried out with a single subcutaneous injection of hCG $(250 \mu \mathrm{g})$. The ovarian double stimulation protocol was similar to the conventional protocol, except triggering was initiated using a GnRH agonist (triptorelin, $0.2 \mathrm{mg}$ ). The patients subjected to dual ovarian stimulation exhibited a statistically significant increase in the mean number of oocytes collected, from 5.3 to 9.3 mature oocytes [33].

Liu et al. [34] conducted a retrospective case-control study involving a total of 116 women aged $\geq 38$ years who were treated with double ovarian stimulation. The women were divided into 4 groups according to the follicular-phase ovarian stimulation protocol: a 
GnRH agonist short protocol, a GnRH antagonist protocol, a mild stimulation protocol, and a progestin pituitary downregulation protocol. The starting dose of gonadotropin was 150-300 IU of FSH, and luteal phase stimulation was performed with $225 \mathrm{IU}$ of hMG daily within 1-3 days of oocyte retrieval. Both instances of triggering were performed using $250 \mathrm{IU}$ of hCG. The numbers of oocytes retrieved and metaphase II oocytes ( $p=0.002$ and $p=0.003$, respectively), fertilized oocytes $(p=0.003)$, cleaved embryos $(p=0.002)$, and top-quality embryos $(p=0.031)$ with luteal-phase stimulation were higher than those retrieved with follicular-phase stimulation [34].

The first study that investigated stimulation with an identical protocol in the follicular and luteal phases of the same menstrual cycle found a similar number of euploid blastocysts in patients with reduced ovarian response. The evidence of multiple follicular waves during a single menstrual cycle in women with diminished ovarian reserve raised important implications. In particular, it indicated that random-start $\mathrm{COH}$ offers an efficient strategy for fertility preservation in young cancer survivors to save time before anticancer therapies. Dual stimulation can maximize the number of oocytes obtained per menstrual cycle and in turn increases the chance of obtaining reproductively competent embryos in the shortest possible time. Randomized controlled trials comparing pregnancy outcomes after dual stimulation are eagerly awaited [35].

In a retrospective observational study that involved 50 cycles in 34 patients who underwent fertility preservation due to breast cancer, follicular-phase or luteal-phase ovarian stimulation with an Al was performed. The numbers of oocytes retrieved at the first and second oocyte pickup did not differ significantly between conventional stimulation and dual stimulation. The researchers demonstrated the efficacy of luteal-phase stimulation and dual stimulation with the $\mathrm{Al}$ protocol among breast cancer patients in regard to increasing the number of oocytes harvested within the limited time available before the initiation of cancer treatment [36].

\section{Triggering with GnRHa versus hCG}

Reddy et al. [37] compared the cycle outcomes and the incidence of ovarian hyperstimulation syndrome (OHSS) when oocyte maturation was triggered by a GnRHa versus hCG in breast cancer patients undergoing fertility preservation. A total of 129 women aged $\leq 45$ years, diagnosed with stage $\leq 3$ breast cancer, and with normal ovarian reserve who desired fertility preservation were compared in the retrospective cohort study. $\mathrm{COH}$ was achieved utilizing letrozole and gonadotropins. Serum anti-Müllerian hormone (AMH) levels were similar between the GnRHa and hCG groups ( $2.7 \pm 1.9$ vs. $2.1 \pm 1.8$ $\mathrm{ng} / \mathrm{mL}$, respectively; $p=0.327$ ). One case of mild or moderate OHSS was reported in the GnRHa group compared to 12 in the hCG group
( $2.1 \%$ vs. $14.4 \%$, respectively; $p=0.032$ ). The maturation and fertilization rates and the number of cryopreserved embryos were significantly higher in the GnRHa group. Therefore, the researchers suggested that the GnRHa trigger improved cycle outcomes as evidenced by the number of mature oocytes and cryopreserved embryos, while significantly reducing the risk of OHSS in breast cancer patients undergoing fertility preservation [37].

A GnRHa trigger can be effective in the induction of oocyte maturation and prevention of OHSS on IVF cycles using an antagonist protocol. Yilmaz et al. [38] reported the comparison of different triggers in different cycles of the same patients. The authors reasoned that the GnRHa trigger is clinically more successful than hCG with regard to OHSS prevention and reproductive outcomes on fresh IVF/ intracytoplasmic sperm injection cycles, although more extensive studies are needed to draw firm conclusions.

In a recent retrospective study by Lin et al. [39], a total of 427 completed GnRHa-downregulated IVF cycles with fresh ET were enrolled. The results of that study suggested that dual triggering of final oocyte maturation with GnRHa and a standard dose of hCG can significantly improve live birth rates, clinical pregnancy rates, and the fertilization rate in women with diminished ovarian reserve undergoing GnRHa-downregulated IVF-intracytoplasmic sperm injection cycles. Clinical results of letrozole-COH efficiency in terms of oocyte quality and pregnancy outcomes in breast cancer patients are still limited, and large data sets will probably be available in several years. The first evaluation of the impact of letrozole- $\mathrm{COH}$ on the oocyte microenvironment in patients with breast cancer has suggested that a GnRHa trigger may improve oocyte quality [40].

However, Asada et al. [41] reported that the usual dose of $\mathrm{GnRHa}$ could fail to trigger oocyte maturation. They suggested that this finding may be attributable to a suboptimal endogenous LH surge due to the severe downregulation of the hypothalamus-pituitary axis. The researchers recommended that a rescue protocol entailing retriggering oocyte maturation using hCG could produce a successful outcome. Chen et al. [42] published a systemic review and meta-analysis that indicated comparable or significantly improved outcomes with the use of GnRH agonists in addition to hCG compared with hCG alone for the triggering of final oocyte maturation.

\section{$B R C A$ gene mutations and ovarian reserve}

$\mathrm{AMH}$ is a sensitive serum marker that approximates the primordial follicle reserve. Titus et al. [43] compared serum AMH concentrations in young breast cancer patients $(n=84)$ with $(n=24$ per group; mean age, $34.8 \pm 4.8$ years) and without ( $n=60$ per group; mean age, $36.3 \pm 3.5$ years) $B R C A$ mutations. The patients with mutations displayed significantly lower serum concentrations of $\mathrm{AMH}$ than the 
patients without them $(1.22 \pm 0.92 \mathrm{ng} / \mathrm{mL}$ vs. $2.23 \pm 1.56 \mathrm{ng} / \mathrm{mL}$, respectively; $p<0.001)$. When the researchers analyzed the impact of $B R C A 1$ versus $B R C A 2$ mutations on ovarian reserve compared to those who tested negative for the same mutations ( $n=60$ per group; mean age, $36.3 \pm 3.5$ years), the significance remained for $B R C A 1 \mathrm{mu}$ tations ( $\mathrm{n}=15$ per group; two patients had both $B R C A 1$ and $B R C A 2$ mutations; mean AMH concentration, $1.12 \pm 0.73 \mathrm{ng} / \mathrm{mL} ; p<0.001)$ but not for $B R C A 2$-only mutations ( $\mathrm{n}=9$ per group; mean $\mathrm{AMH}$ concentration, $1.39 \pm 1.20 ; p=0.127)$. The researchers used transgenic mouse models to confirm that women with BRCA1 mutations had diminished ovarian reserve. The data are also consistent with the decreased response to ovarian stimulation and earlier menopausal age shown previously in human BRCA1 mutation carriers [43].

Recently, Peccatori et al. [44] suggested that individuals with $B R C A 1$ mutations have significantly lower mature oocyte yields. This independence of the role of BRCA1 mutations was confirmed by the comparison of this study group with a group of patients without $B R C A$ mutations who were being treated for breast cancer. Oocyte cryopreservation is a feasible option that should be suggested to these patients, especially considering the frequent recommendation of prophylactic bilateral salpingo-oophorectomy at the age of 40 for both women with BRCA1 mutations and those with BRCA2 mutations.

In contrast, a retrospective cohort study demonstrated that $B R C A$ carriers with and without malignancy had comparable ovarian reserve and response to ovarian stimulation to noncarriers undergoing fertility preservation [45]. However, data from Korean patients indicated that breast cancer patients with BRCA mutations had significantly lower serum AMH levels, although no significant differences were found between those with BRCA1 and those with BRCA2 mutations. The researchers recommended that fertility preservation be considered more aggressively in young breast cancer patients with BRCA mutations [46].
Winship et al. [47] published the protocol for a prospective observational study that will be the first large-scale study aimed at exploring the measurement of ovarian reserve in both $B R C A 1$ and $B R C A 2$ mutation carriers compared with age-matched patients without these mutations. The results of this study have the potential to clarify the association between circulating $\mathrm{AMH}$ and follicle density and thus help elucidate the mechanism of oocyte loss during reproductive life, particularly for women who are thought to have reduced ovarian reserve, such as $B R C A$ mutation carriers.

Women with $B R C A$ mutations (in consideration of the differences between $B R C A 1$ and $B R C A 2$ mutations) may be informed about the possible advantages of oocyte cryopreservation, along with the general risks, cost, and effectiveness of the procedure. Putting aside the unresolved debate on the impact of BRCA mutations on ovarian reserve, a personalized assessment of serum $\mathrm{AMH}$ or antral follicle count at the time of counseling can also be useful. Based on current knowledge, standardized clinical management cannot be recommended. However, even if a policy of fertility preservation in young, healthy $B R C A$ carriers cannot be advocated across the board, it deserves consideration and should be carefully discussed on an individual basis [48].

Further research on the impact of $B R C A$ mutations on fertility is necessary to confirm these findings and provide a better understanding of the underlying pathophysiology. Given that conflicting evidence exists regarding diminished fertility in this specific population, larger prospective trials are required to clarify the impact of $B R C A$ mutations on ovarian aging.

\section{Conclusion}

In Korea, requests for fertility preservation treatment in breast cancer patients are becoming increasingly common. Random-start $\mathrm{COH}$ and dual ovarian stimulation with the co-administration of letrozole

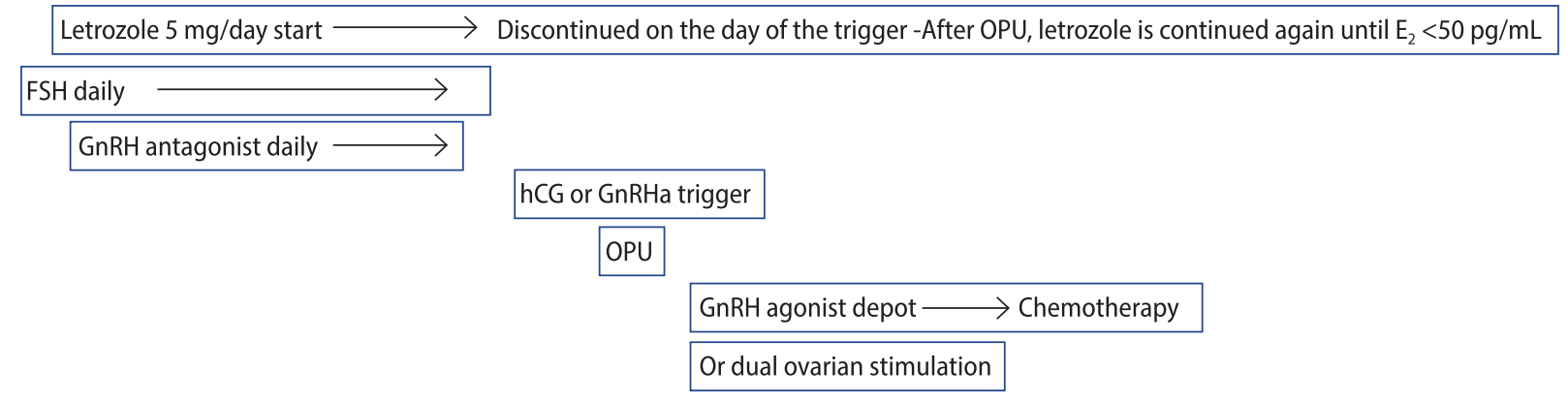

$1-2-3$ $12-14$ -- Days of random start

Figure 1. Controlled ovarian hyperstimulation protocol for fertility preservation in women with breast cancer. OPU, ovum pick-up; $E_{2}$, estradiol; FSH, follicle-stimulating hormone; GnRH, gonadotropin-releasing hormone; hCG, human chorionic gonadotropin; $\mathrm{GnRHa}$, gonadotropin-releasing hormone agonist. 
are feasible for the efficient harvest of oocytes or embryos and the reduction of the total duration of fertility preservation treatment, and these techniques work without raising peak estradiol levels and correspondingly increasing breast cancer risk. The use of a GnRHa trigger may improve cycle outcomes in breast cancer patients undergoing fertility preservation. In young breast cancer patients with $B R C A$ mutations, particularly BRCA1 mutations, more effective fertility preservation treatment should be considered. The $\mathrm{COH}$ protocol for fertility preservation in women with breast cancer is outlined in Figure 1. Additional clinical studies with larger samples are required to confirm the optimal protocol for fertility preservation in young women with breast cancer.

\section{Conflict of interest}

No potential conflict of interest relevant to this article was reported.

\section{ORCID}

\section{So Yun Park \\ Kyungah Jeong \\ Eun Hye Cho \\ Hye Won Chung}

\section{Author contributions}

Conceptualization: KJ. Writing-original draft: KJ, SYP, EHC. Writingreview \& editing: KJ, HWC.

\section{References}

1. World Health Organization. GLOBOCAN 2012: estimated cancer incidence, mortality and prevalence worldwide in 2012 [Internet]. Lyon: World Health Organization International Agency for Research on Cancer; 2012 [cited 2020 Oct 29]. Available from: http:// globocan.iarc.fr/ Pages/fact_sheets_cancer.aspx.

2. Ruddy KJ, Gelber SI, Tamimi RM, Ginsburg ES, Schapira L, Come $\mathrm{SE}$, et al. Prospective study of fertility concerns and preservation strategies in young women with breast cancer. J Clin Oncol 2014; 32:1151-6.

3. Ganz PA, Bower JE, Stanton AL. Special issues in younger women with breast cancer. Adv Exp Med Biol 2015;862:9-21.

4. National Cancer Institute. SEER cancer statistics review (CSR) 1975-2012: cancer of the female breast (invasive) [Internet]. Bethesda: National Cancer Institute; 2015 [cited 2020 Oct 29]. Available from: http://seer.cancer.gov/csr/ 1975_2012/browse_
csr.php?sectionSEL = 4\&pageSEL = sect_04_table.14.html.

5. Ko BS, Noh WC, Kang SS, Park BW, Kang EY, Paik NS, et al. Changing patterns in the clinical characteristics of Korean breast cancer from 1996-2010 using an online nationwide breast cancer database. J Breast Cancer 2012;15:393-400.

6. Kim H, Kim SK, Lee JR, Hwang KJ, Suh CS, Kim SH. Fertility preservation for patients with breast cancer: The Korean Society for Fertility Preservation clinical guidelines. Clin Exp Reprod Med 2017; 44:181-6.

7. Advani P, Moreno-Aspitia A. Current strategies for the prevention of breast cancer. Breast Cancer (Dove Med Press) 2014;6:59-71.

8. Zhou W, Slingerland JM. Links between oestrogen receptor activation and proteolysis: relevance to hormone-regulated cancer therapy. Nat Rev Cancer 2014;14:26-38.

9. Nilsson S, Gustafsson JA. Estrogen receptor action. Crit Rev Eukaryot Gene Expr 2002;12:237-57.

10. Huang C, Wang X, Sun B, Li M, Zhao X, Gu Y, et al. Study on mouse model of triple-negative breast cancer: association between higher parity and triple-negative breast cancer. Target Oncol 2015;10:85-97.

11. Palmer JR, Boggs DA, Wise LA, Ambrosone CB, Adams-Campbell $L L$, Rosenberg $L$. Parity and lactation in relation to estrogen receptor negative breast cancer in African American women. Cancer Epidemiol Biomarkers Prev 2011;20:1883-91.

12. Shim YJ, Seol A, Lee D, Kim SK, Lee JR, Jee BC, et al. The serum estradiol/oocyte ratio in patients with breast cancer undergoing ovarian stimulation with letrozole and gonadotropins. Obstet Gynecol Sci 2018;61:242-6.

13. Turan V, Bedoschi G, Emirdar V, Moy F, Oktay K. Ovarian stimulation in patients with cancer: impact of letrozole and BRCA mutations on fertility preservation cycle outcomes. Reprod Sci 2018; 25:26-32.

14. Oktay K, Buyuk E, Libertella N, Akar M, Rosenwaks Z. Fertility preservation in breast cancer patients: a prospective controlled comparison of ovarian stimulation with tamoxifen and letrozole for embryo cryopreservation. J Clin Oncol 2005;23:4347-53.

15. Oktay K, Buyuk E, Rodriguez-Wallberg KA, Sahin G. In vitro maturation improves oocyte or embryo cryopreservation outcome in breast cancer patients undergoing ovarian stimulation for fertility preservation. Reprod Biomed Online 2010;20:634-8.

16. Oktay K, Hourvitz A, Sahin G, Oktem O, Safro B, Cil A, et al. Letrozole reduces estrogen and gonadotropin exposure in women with breast cancer undergoing ovarian stimulation before chemotherapy. J Clin Endocrinol Metab 2006;91:3885-90.

17. Domingo J, Guillen V, Ayllon Y, Martinez M, Munoz E, Pellicer A, et al. Ovarian response to controlled ovarian hyperstimulation in cancer patients is diminished even before oncological treatment. 
Fertil Steril 2012;97:930-4.

18. Revelli A, Porcu E, Levi Setti PE, Delle Piane L, Merlo DF, Anserini P. Is letrozole needed for controlled ovarian stimulation in patients with estrogen receptor-positive breast cancer? Gynecol Endocrinol 2013;29:993-6.

19. Azim AA, Costantini-Ferrando M, Lostritto K, Oktay K. Relative potencies of anastrozole and letrozole to suppress estradiol in breast cancer patients undergoing ovarian stimulation before in vitro fertilization. J Clin Endocrinol Metab 2007;92:2197-200.

20. Checa Vizcaino MA, Corchado AR, Cuadri ME, Comadran MG, Brassesco M, Carreras R. The effects of letrozole on ovarian stimulation for fertility preservation in cancer-affected women. Reprod Biomed Online 2012;24:606-10.

21. Domingo J, Garcia-Velasco JA. Oocyte cryopreservation for fertility preservation in women with cancer. Curr Opin Endocrinol Diabetes Obes 2016;23:465-9.

22. Rodgers RJ, Reid GD, Koch J, Deans R, Ledger WL, Friedlander M, et al. The safety and efficacy of controlled ovarian hyperstimulation for fertility preservation in women with early breast cancer: a systematic review. Hum Reprod 2017;32:1033-45.

23. Kim J, Turan V, Oktay K. Long-term safety of letrozole and gonadotropin stimulation for fertility preservation in women with breast cancer. J Clin Endocrinol Metab 2016;101:1364-71.

24. Meirow D, Raanani H, Maman E, Paluch-Shimon S, Shapira M, Cohen Y, et al. Tamoxifen co-administration during controlled ovarian hyperstimulation for in vitro fertilization in breast cancer patients increases the safety of fertility-preservation treatment strategies. Fertil Steril 2014;102:488-95.

25. Cakmak H, Rosen MP. Random-start ovarian stimulation in patients with cancer. Curr Opin Obstet Gynecol 2015;27:215-21.

26. Cakmak H, Tran ND, Zamah AM, Cedars MI, Rosen MP. A novel "delayed start" protocol with gonadotropin-releasing hormone antagonist improves outcomes in poor responders. Fertil Steril 2014;101:1308-14.

27. Cakmak H, Katz A, Cedars MI, Rosen MP. Effective method for emergency fertility preservation: random-start controlled ovarian stimulation. Fertil Steril 2013;100:1673-80.

28. Martinez F, Clua E, Devesa M, Rodriguez I, Arroyo G, Gonzalez C, et al. Comparison of starting ovarian stimulation on day 2 versus day 15 of the menstrual cycle in the same oocyte donor and pregnancy rates among the corresponding recipients of vitrified oocytes. Fertil Steril 2014;102:1307-11.

29. Danis RB, Pereira N, Elias RT. Random start ovarian stimulation for oocyte or embryo cryopreservation in women desiring fertility preservation prior to gonadotoxic cancer therapy. Curr Pharm Biotechnol 2017;18:609-13.

30. Kuang Y, Chen Q, Hong Q, Lyu Q, Ai A, Fu Y, et al. Double stimula- tions during the follicular and luteal phases of poor responders in IVF/ICSI programmes (Shanghai protocol). Reprod Biomed Online 2014;29:684-91.

31. Ubaldi FM, Capalbo A, Vaiarelli A, Cimadomo D, Colamaria S, Alviggi $C$, et al. Follicular versus luteal phase ovarian stimulation during the same menstrual cycle (DuoStim) in a reduced ovarian reserve population results in a similar euploid blastocyst formation rate: new insight in ovarian reserve exploitation. Fertil Steril 2016;105:1488-95.

32. Moffat R, Pirtea P, Gayet V, Wolf JP, Chapron C, de Ziegler D. Dual ovarian stimulation is a new viable option for enhancing the oocyte yield when the time for assisted reproductive technnology is limited. Reprod Biomed Online 2014;29:659-61.

33. Cardoso MC, Evangelista A, Sartorio C, Vaz G, Werneck CL, Guimaraes FM, et al. Can ovarian double-stimulation in the same menstrual cycle improve IVF outcomes? JBRA Assist Reprod 2017; 21:217-21.

34. Liu C, Jiang H, Zhang W, Yin H. Double ovarian stimulation during the follicular and luteal phase in women $\geq 38$ years: a retrospective case-control study. Reprod Biomed Online 2017;35:678-84.

35. Vaiarelli A, Venturella R, Vizziello D, Bulletti F, Ubaldi FM. Dual ovarian stimulation and random start in assisted reproductive technologies: from ovarian biology to clinical application. Curr Opin Obstet Gynecol 2017;29:153-9.

36. Nakasuji T, Kawai K, Ishikawa T, Teraoka K, Takeuchi S, Miyagawa T, et al. Random-start ovarian stimulation with aromatase inhibitor for fertility preservation in women with Japanese breast cancer. Reprod Med Biol 2019;18:167-72.

37. Reddy J, Turan V, Bedoschi G, Moy F, Oktay K. Triggering final oocyte maturation with gonadotropin-releasing hormone agonist $(\mathrm{GnRHa})$ versus human chorionic gonadotropin (hCG) in breast cancer patients undergoing fertility preservation: an extended experience. J Assist Reprod Genet 2014;31:927-32.

38. Yilmaz N, Ceran MU, Ugurlu EN, Gulerman HC, Engin Ustun Y. GnRH agonist versus HCG triggering in different IVF/ICSI cycles of same patients: a retrospective study. J Obstet Gynaecol 2020;40: 837-42.

39. Lin MH, Wu FS, Hwu YM, Lee RK, Li RS, Li SH. Dual trigger with gonadotropin releasing hormone agonist and human chorionic gonadotropin significantly improves live birth rate for women with diminished ovarian reserve. Reprod Biol Endocrinol 2019;17:7.

40. Goldrat O, Van Den Steen G, Gonzalez-Merino E, Dechene J, Gervy C, Delbaere A, et al. Letrozole-associated controlled ovarian hyperstimulation in breast cancer patients versus conventional controlled ovarian hyperstimulation in infertile patients: assessment of oocyte quality related biomarkers. Reprod Biol Endocrinol 2019;17:3. 
41. Asada Y, Itoi F, Honnma H, Takiguchi S, Fukunaga N, Hashiba Y, et al. Failure of GnRH agonist-triggered oocyte maturation: its cause and management. J Assist Reprod Genet 2013;30:581-5.

42. Chen CH, Tzeng CR, Wang PH, Liu WM, Chang HY, Chen HH, et al. Dual triggering with $\mathrm{GnRH}$ agonist plus hCG versus triggering with hCG alone for IVF/ICSI outcome in GnRH antagonist cycles: a systematic review and meta-analysis. Arch Gynecol Obstet 2018; 298:17-26.

43. Titus S, Li F, Stobezki R, Akula K, Unsal E, Jeong K, et al. Impairment of BRCA1-related DNA double-strand break repair leads to ovarian aging in mice and humans. Sci Transl Med 2013;5:172ra21.

44. Peccatori FA, Mangili G, Bergamini A, Filippi F, Martinelli F, Ferrari F, et al. Fertility preservation in women harboring deleterious BRCA mutations: ready for prime time? Hum Reprod 2018;33:181-7.

45. Gunnala V, Fields J, Irani M, D'Angelo D, Xu K, Schattman G, et al.
BRCA carriers have similar reproductive potential at baseline to noncarriers: comparisons in cancer and cancer-free cohorts undergoing fertility preservation. Fertil Steril 2019;111:363-71.

46. Son KA, Lee DY, Choi D. Association of BRCA mutations and anti-Müllerian hormone level in young breast cancer patients. Front Endocrinol (Lausanne) 2019;10:235.

47. Winship AL, Willson C, Hansen KR, Hutt KJ, Hickey M. Do BRCA1 and BRCA2 gene mutation carriers have a reduced ovarian reserve? Protocol for a prospective observational study. BMJ Open 2019;9:e033810.

48. Porcu E, Cillo GM, Cipriani L, Sacilotto F, Notarangelo L, Damiano G, et al. Impact of BRCA1 and BRCA2 mutations on ovarian reserve and fertility preservation outcomes in young women with breast cancer. J Assist Reprod Genet 2020;37:709-15. 